Radiologe 2013 · 53:302

DOI 10.1007/s00117-012-2415-z

Online publiziert: 4. April 2013

(c) Springer-Verlag Berlin Heidelberg 2013
C. Herold ${ }^{1} \cdot$ M.F. Reiser ${ }^{2} \cdot$ A. Graser ${ }^{2}$

${ }^{1}$ Universitätsklinik für Radiodiagnostik, Medizinische Universität Wien, Wien

${ }^{2}$ Institut für Klinische Radiologie, Klinikum der

Ludwig-Maximilians-Universität München, Campus Großhadern, München

\title{
Onkologische Bildgebung
}

Sehr geehrte Kolleginnen und Kollegen,

kein Gebiet der diagnostischen und interventionellen Radiologie entwickelt sich derzeit so dynamisch wie die Bildgebung bei malignen Erkrankungen. Der wachsenden Bedeutung der „onkologischen Bildgebung" wurde jüngst international in der European Society of Radiology (ESR) durch die Gründung der European Society of Oncologic Imaging (ESOI) Rechnung getragen, in der Deutschen Röntgengesellschaft (DRG) steht die Gründung der Arbeitsgruppe Onkologische Bildgebung unmittelbar bevor. Hintergrund dieser Bemühungen ist es, eine Verbesserung der radiologischen Befundqualität durch ein breites Fortbildungsangebot und standardisierte Ausbildung der Kollegen zu erreichen. Ferner sollen sie die Kooperation stärken zwischen Radiologen und zuweisenden Kollegen aus Onkologie bzw. onkologischer Chirurgie sowie den onkologisch tätigen Urologen und Gynäkologen, um nur einige Beispiele zu nennen. Somit passt das hier vorliegende Themenheft sehr gut in die Landschaft einer geschärften Wahrnehmung der besonderen Bedeutung der bildgebenden Disziplinen bei Diagnostik und Behandlung von Patienten mit malignen Erkrankungen.

Um mit der rapiden Entwicklung bei chirurgischen Techniken, modernen zielgerichteten Therapien und systemischen Kombinationstherapien Schritt zu halten, müssen Radiologen Kenntnisse über spezifische bildmorphologische Veränderungen erwerben, die infolge von und während dieser Therapien zu erwarten sind. Zudem ist das Erkennen therapieassozi- ierter Nebenwirkungen, die charakteristische Bildbefunde erzeugen, von Bedeutung. Eine weitere Anforderung an den onkologisch tätigen Radiologen ist die exakte Kenntnis verschiedener kriterienbasierter Befundungssysteme, wie RECIST-, Choi- und Cheson-Kriterien. Diese sind nicht nur in klinischen Studien anzuwenden, sondern können auch als Grundstruktur für die klinische Befunderhebung dienen, da in ihnen eine standardisierte Anleitung zur exakten Beurteilung eines Therapieansprechens festgehalten ist.

Letztendlich hat dieses Heft also zweierlei Zielsetzungen: zu informieren und fortzubilden. Sie werden bei seiner Lektüre Informationen über die Bedeutung von Tumorboards ebenso finden wie eine strukturierte Übersicht über therapieassoziierte Nebenwirkungen und deren Visualisierung sowie kriterienbasierte Befundung. Zudem haben wir Beiträge zum Thema Begriffe und Definitionen in der onkologischen Bildgebung sowie zu den Indikationen und Limitationen der Schnittbildverfahren CT, MRT und PET/ CT ausgewählt.

Wir hoffen sehr, dass Sie Freude bei der Lektüre der hier vorliegenden Arbeiten haben, die Ihnen die onkologische Bildgebung als Ganzes näherbringen und $\mathrm{Ihr}$ Wissen auf diesem Gebiet vertiefen sollen.

Wie in allen Gebieten der Medizin steht das Wohl der Patienten im Mittelpunkt. Die interdisziplinäre Zusammenarbeit spielt gerade bei onkologischen $\mathrm{Pa}$ tienten eine herausragende Rolle. Dabei ist der kompetente diagnostische und interventionelle Radiologe ein geschätzter Partner, der maßgeblich dazu beitra- gen kann, dass die für den jeweiligen Patienten am besten geeignete Therapie gewählt wird.

Ihre

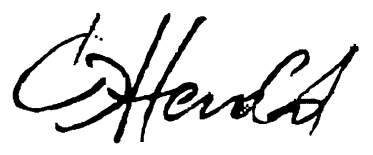

Prof. Dr. Christian Herold

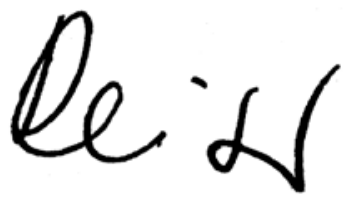

Prof. Dr. Dr. h. c. Maximilian F. Reiser

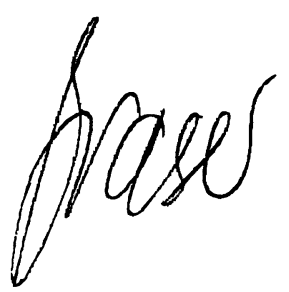

PD Dr. Arno Graser

\section{Korrespondenzadresse}

Univ.-Prof. Dr. C. Herold

Universitätsklinik für Radiodiagnostik,

Medizinische Universität Wien,

Währinger Gürtel 18-20, 1090 Wien,

Österreich

Christian.Herold@akhwien.at 